\title{
Innovations financières et stabilité du system financier : le cas des crédits default swap (CDS).
}

\begin{abstract}
:
Credits default swaps (CDS) are financial innovations that have experienced a fuliginous development since their appearance for the first time in 1997; they are used in the transfer of credit risks especially in bonds markets, and more recently in the process of synthetic securitization. Located equally between insurance and financial product, the CDS are negotiated hedging contracts using as underlying a portfolio of loans or bonds, and negotiated by Monoline in an unorganized market.The CDS are considered by experts as one of the main aggravating factors of the subprime crisis (2007) and the global financial crisis (2008) that has followed. These same innovative financial products have been widely used by speculators during the crisis of Greek Sovereign Debt (2010), which contributed to a wide spreading crisis to other EU countries and gave it in the process its systemic character. The pernicious effect of unnecessary financial innovations on the stability of the financial system has been set forth by many researchers, including the precursor Hyman Minsky (1992).

Keywords: Financial Innovation, Credit default Swap, Financial Stability, Synthetic Securitization, Financial Crisis.
\end{abstract}

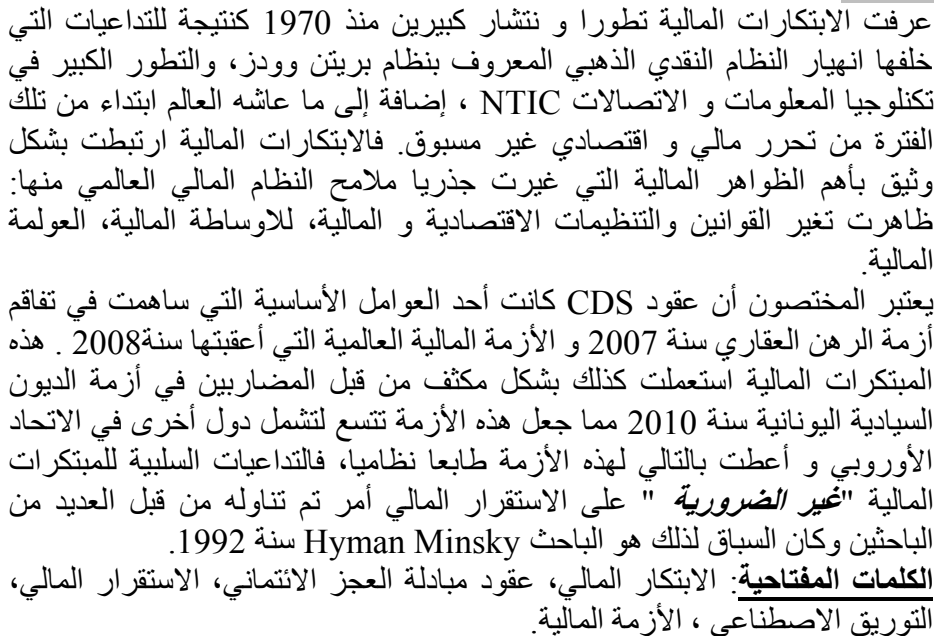

Dr. Bennamoun Hamadou

Faculté sciences Economique

Université Abdelhamid Mehri

Constantine2

\begin{abstract}
Introduction :
Les innovations financières ont connu un rapide développement depuis les années 1970, favorisées pas l'effondrement du système monaitaire de breton woods, l'essor des NTIC et le début d'une vague de libéralisme économique et financier débridé. Les innovations financières ont joué un rôle décisif dans la mutation du
\end{abstract}


système financier, car elles sont intimement liées aux principales évolutions qui ont transformés en profondeur la sphère financière mondiale ; à savoir la dérèglementation financière, la désintermédiation et la globalisation financière.

Pour certains l'innovation financière est une source majeure de croissance économique, grâce à la diversification et l'intensification des moyens et sources de financement à destination de l'économie et la multiplication des produits et techniques de couverture des risques, ce qui constitue en même temps des puissants facteurs de croissance des marches financiers. Pour d'autres l'innovation financière rime avec instabilité car d'après eux elle incite généralement à une prise de risque excessive, lies à l'immense effet levier qu'elle autorise, sans oublier son rôle dans accroissement de la complexité et l'opacité des systèmes financiers.

Le développement phénoménal de la titrisation et des produits dérivés ces dernières années a donné naissance à une multitude de produits financiers innovants, parmi ces produits (et non des moindres) il y a les contrats CDS (crédit default swap) donc le volume des encoures a atteint des sommets les années qui ont précédées la crise financière de 2008( plus de 6 trillion de dollars), avant de connaitre un net recul suite à l'effondrent de plusieurs institution financières de grande envergure qui jouées le rôle d'assureurs monoline. De là il devient légitime de poser la question suivante : quelle est la relation entre l'expansion des innovations financières et la stabilité du system financier?

\section{1- Définition de l'innovation Financière :}

L'innovation financière se définit comme la création ou la modification d'un produit, un service ou un marché financier. Les innovations financières constituent un ensemble vaste et hétérogène dans lequel il convient de distinguer les innovations radicales des innovations incrémentales, une très large part des innovations est de nature incrémentale, car le plus souvent, les innovations financières sont obtenues grâce à une simple modification à la marge de certaines caractéristiques du produit initiale. (1)

C'est la création de nouveaux instruments financier à partir de produits financiers existants, elle s'apparente a une «mutation biologique ou génétique " qui débouche sur un élément hybride, mutant ou innovant. Certains chercheures qualifient l'innovation financière de "gène récessif", car de nombreuses innovations financières existaient déjà, sous une forme ou sous une autre bien avant qu'elles n'apparaissent a la faveur d'un changement de l'environnement économique et financier. (2)

2-Classification des innovations financières:

La distinction entre les différentes classes ou types d'innovations financières est un travail très délicat compte tenu de leur nombre et de leurs 
complexité et la difficulté à faire la distinction entre les différents produits financiers innovants. L'emprunt du cadre conceptuel de l'économie industrielle a permis de nombreuses avancées dans la connaissance des phénomènes de l'innovation financière.

Selon l'approche désormais classique de Joseph Schumpeter (1911), fondateur des théories du progrès technique, l'innovation est simplement définie comme de «nouvelles combinaisons productives» qui peuvent être de cinq ordres dans sa célèbre classification: (3)

1- la fabrication d'un bien nouveau ou d'une qualité nouvelle d'un bien non familière aux

Consommateurs.

2- l'introduction d'une méthode de production nouvelle, c'est-à-dire, un procédé de fabrication inconnu dans la branche au- par- avant.

3- l'ouverture d'un débouché nouveau, c'est à dire d'un marché sur lequel la branche

Intéressée n'était jusqu'alors pas présente.

4- la conquête d'une nouvelle source de matières premières ou de produits semi ouvrés.

5- la réalisation d'une nouvelle organisation pour la firme intéressée.

Transposée à l'analyse de l'innovation financière la précédente classification permet de faire la distinction suivante :

1- Innovation de processus : généralement c'est l'introduction de la technologie (NTIC) a l'univers des marches financiers (la cotation électronique, compensation électronique, moyens de payements électronique, internet, Swift.....) .

2- Innovation de produit: apparition de nouveaux produits financiers, la plupart du temps grâce à la modification des caractéristiques des produits existants (TCN, OC, OBSA, OBSO, OPTION, SWAP, COLAR.....).

3- Innovation de Marché : La création d'un nouveau marché financier (Nasdaq, Monep, les marchés à terme, Euro stock......).

4- Innovation de matière première : consistant en une nouvelle forme ou produit pour la collecte de l'épargne (NOW, Super NOW, livret d'épargne....).

5- Innovation en matière d'organisation: de nouvelles formes d'organisation (la bancassurance, les holdings financier, Le shadow Banking.....).

\section{3- Les specifités de l'innovation financière :}

La particularité de l'innovation financière, comparé aux autre types d'innovations, réside dans : . (4)

1- Le caractère hybride, car la plus part des innovations sont le résultat de plusieurs innovations en même temps (Les produits dérivés, les TRAKERS, TCN...). 
2- La non brevetabilité due à la difficulté de protéger le secret de production relatif à ces produits, la raison étant que les innovations ne sont en fait que «des contrats » facilement imitables.

3- L'innovation financière disparait rarement, car elle a pour conséquence généralement d'ajouter un produit a la gamme de produit déjà existant et qui continu d'exister.

4- L'innovation financière se diffuse aisément d'une place financière à une autre facilité par le bais du phénomène de globalisation financière.

5- L'innovation financière naît la plus part du temps aux USA, ou l'activité financière est très dynamique, ce pays représente le creuset de l'innovation financière car elle est le fait du secteur privé, elle se propage par la suite au reste du monde par imitation et capillarité, spécialement en l'Europe ou l'innovation financières et en majorité d'origine publique. (5)

\section{4- Les raisons d'être de l'innovation financière :}

Deux raisons majeures peuvent nous aider à expliquer le déclanchement et l'essor de l'innovation financière : La demande et l'offre.

a) Modification de la demande: un changement profond de l'environnement économique peut être à l'origine d'un changement de la demande des produits. La volatilité des taux intérêt et l'inflation galopante, qui ont suivis l'abandon du système de Bretton Wood's en1971, ont exacerbé les incertitudes des investisseurs quant au rendement des investissements qui se orientés vers des produits financiers qui pourraient diminuer le risque et augmenter le rendement (6).

Toutefois, il faut préciser que dans le cas où la demande est à l'origine de l'innovation financière, il en reste pas moins que les investisseurs demandent des «caractéristiques » et non pas les actifs financiers eux-mêmes, à partir de là, les institutions financières entre dans une phase de mise au point de produits financiers innovants sur la base des caractéristiques demandées ( ex : les taux d'intérêt variables produits dérivés, produits d'épargne.......). Cette adaptation du produit financier à une modification d'une caractéristique de la demande, ellemême liée une nouvelle conjecture, correspond à une nouvelle innovation financière. (7)

b) Modification de l'offre : l'autre moteur de l'innovation financière c'est la modification de l'offre, cette approche est qualifier d'approche par l'offre, puisque ce sont les institutions financières qui sont à l'origine des innovations financières. Vu de cet angle nous pouvons résumer les raisons qui poussent les institutions financières à innover comme suit :

$\checkmark$ Consolider la position concurrentielle et gagne des parts de marché: L'innovation financière est pour les institutions financières comme une nécessaire adaptation dans un 
environnement changent et de plus en plus exigent, ils doivent aujourd'hui fournir de l'information fiable plus rapidement, prendre des décisions plus vite, surveiller la concurrence et être à l'affut de nouvelles façons de servir les clients. Ils doivent être plus comptants plus imaginatifs et disposer d'équipements high Tech (8). A travers l'innovation financière les intermédiaires financiers essaye de fidéliser la clientèle, et maintenir une image de marque favorable associée à un établissement considéré comme innovant.

$\checkmark$ L'apparition de nouveaux canaux d'accès a l'offre de produit financier: La percée fulgurante de l'informatique et des moyens de communication (NTIC) constituent un puissant vecteur de l'innovation financière. La dématérialisation des transactions a contribué à amplifier la circulation de l'information, et par la même occasion, la capacité des marche financiers, elle a offre à tous les agents qui le souhaitent la possibilité de se présenter directement sur le marché face aux investisseurs potentiels. (ex : les on-line discounts brokers aux USA, le e-Banking.....).Il devint ainsi claire que l'innovation financière n'est pas générée par la seule modification de l'offre, mais aussi par les canaux d'accès a cette offre de produits financiers. (9)

$\checkmark$ La volonté de s'affranchir des contraintes règlementaires: l'innovation financière s'explique aussi par les stratégies antagonistes de deux catégories d'agents, la puissance publique d'un cote et les intermédiaires financiers de l'autre. Ces derniers mettent en place des stratégies de contournement de la règlementation en place qu'ils jugent excessives ou contraignante. (10)

L'innovation financière peut donc avoir pour but de contourner les contraintes réglementaires, c'est le système connu de "la dialectique de la réglementation » qui commence par une première phase de réglementation, qui s'accompagne dans un deuxième temps de mouvement de contournement de cette réglementation, par le bais d'innovations financières, et qui peut être ensuite suivie de mouvement de déréglementation de réréglementation (11).

A ce titre, la naissance des euro dollars et du marché de l'euro dollars au début des années 70, fut une opportunité offerte aux banques américaines afin de d'augmenter les taux d'intérêts sur les dépôts et de contourner la réglementation, dite « $\mathrm{Q}$ Act » de 1933, qui plafonné les taux d'intérêt rémunérant les comptes 
de dépôts. Un autre exemple c'est le développement phénoménale des activités de marche du système bancaire, qui n'a été possible que grâce à une innovation financière de taille, à savoir les entités hors bilan ou "Hedge Funds", qui ont permis au banque de contourner la réglementation (Glass Seagalll Act de 1933 aux USA) qui séparé les activités financières.

Mais une autre modification de l'offre de produits financiers a révolutionner le paysage financier spécialement aux USA, c'est la technique de "Titrisation ", développée pas Lewis S.Ranieri un trader travaillant pour la banque d'affaire Salomon Brothers, est qui consiste à convertir des prêts bancaires en titre négociables au moyen d'un montage financier très complexe et l'utilisation de produits financiers innovants (ABS, MBS).(12)

La titrisation est née aux Etats unis dans les années1960, il s'agissait alors de relancer le financement de l'immobilier résidentiel qui se révélait inadapté en raison, d'une part, du niveau élevé des taux d'intérêt, et d'autre part, du plafonnement de la rémunération des dépôts soumis à la règlementation " $\boldsymbol{Q} » .(13)$

A partir les années 1990, le développement très rapide de l'utilisation de la titrisation par les établissements de bancaire s'explique par le fait qu'ils peuvent en retirer plusieurs avantages. Soumises au Ratio Cooke imposé par les accords de Bale 1 de 1988, les établissements de crédits peuvent arbitrer entre des opérations de titrisation et les opérations mobilisant des fonds propres, de plus ce mécanisme peut conduire à un abaissement de cout du crédit. (14)

Le dispositif de Bale fut un succès au plan de l'optimisation des fonds propres, puisqu'il conduit les établissements de crédit à augmenter de façon très significative leurs fonds propres. Mais la sophistication des opérations de titrisation, innovation financière imaginée par les banques dans le but de se soustraire aux contraintes règlementaires, la rendu presque obsolète et a nécessité des reformes ultérieures. (15)

$\checkmark$ La tendance des acteurs économiques vers «le risk management $»$ : les perturbations qui ont secoué les économies mondiales au lendemain de l'effondrement du system breton woods en 1971, ont accentué les incertitudes des agents économiques. Pour pallier à cette incertitude, les marches des produits dérivés vont connaitre à partir de cette date un essor considérable, qui s'est amplifié à partir des années 90 spécialement aux USA .Les banques américains détenaient a elles 
seules $40 \%$ des produit dérivés financiers au monde, la majeur partie été échangée sur les marchés d Gré à Gré (OTC) (16).

Sous l'impulsion du développent de ces marchés à terme, une multitude d'innovation financières est venue enrichir la panoplie d'instruments financiers offerts aux investisseurs, qui sont devenus à leur tours très friand de ces nouveaux produits qui leurs permettaient, dans cet environnement devenu très hostile, de transférer une partie des risques a d'autres acteur du marché. Parmi ces produits, qui constitues la majeur partie des innovations financiers dans la monde, nous pouvant citer: (les Swaps, les Future, les options, les Warrants, CDS, CDO, FRA, COLAR, FLOOR, .........).

Le « Risk Management » est devenu un concept clés dans la stratégie des acteurs économiques à partir des années 70 . Le développement de nouveaux instruments de couverture des risques, à partir de cette date, est devenu une tendance lourde initiée par les institutions financières, à tel point que ces mêmes institutions sont désormais tributaires, pour leurs succès, de la bonne maitrise de ces instruments innovants. Le risque manager de nos jours se doit donc d'étoffé continuellement son arsenal d'outils d'identification et de gestion de risque, aussi l'innovation dans ce domaine est essentielle et permanente pour maintenir la performance du système financier, et la confiance du public dans ce système. (17)

\section{5-Innovation Financière et Stabilité Financière:}

Le débat sur l'utilité et les dangers des innovations financières est très riche est très contrasté .Certains défendent l'idée selon laquelle une innovation financière est utile si elle permettrais aux marches financiers d'être plus efficients, ils mettent en garde les investisseurs contre les innovations financières qui n'en sont pas vraiment, dans la mesure ou elles ne contribuent pas à rendre les marchés plus efficients mais permettent, en revanche, à leurs promoteurs de s'enrichir rapidement et abusivement. (18)

L'innovation financière "utile " répond à des imperfections des marchés financiers et permet potentiellement d'accroître leur efficience. L'innovation financières et la conséquence de changements profonds de l'environnement économique représentent le plus souvent une adaptation des méthodes de financement à des changements des structures économiques, et est à ce titre un facteur de croissance de l'économie. Certaines innovations financières (le capital Risk, le capital Investment, les business angles......) ont même facilité le progrès technique dans le sens où elles ont permis le financement de projets innovants qui avaient difficilement accès aux sources de financement 
traditionnelles ( Les Dot.com, Start Up......) : en l'absence d'innovations financières, le développement technologique et économique des sociétés modernes aurait été ralenti. (19)

La vision traditionnelle selon laquelle l'innovation est une source majeure de croissance économique, insiste sur le fait que l'innovation financière a été à l'origine de l'amélioration des sévices bancaires, a facilité le partage des risques entre les différents acteurs économiques (hedgers and speculators), a complété la gamme de produit financiers et ainsi améliorer l'allocation des ressources .L'innovation financière a été, durant les dernières décennies, la vraie force motrice du renforcement du system financier et de la croissance économique. L'émergence des banques d'investissement et des institutions de crédit spécialisées a permis le financement du chemin de fer au 19 siècle, et le "venture capital ou capital risk» a été nécessaire au financement des Start- up et des entreprises du hi-tec au 20 siècle. Enfin les innovations financières ont contribué largement à financer les entreprises de bio- technologie au 21 siècle.

Pour ce qui est des potentielles externalités négatives associées aux innovations financières, on constate que certaines innovations ont opérer un bouleversement d'envergure dans l'activité du système financier. La technique de titrisation, par exemple a changé le modèle d'affaire des banques (originate to hold, originate to distribute), surtout en matière de précautions ex ante que les banques doivent prendre lors de l'examen des demandes de crédits. Les innovations financières incitent generalement à une prise de risque excessive due au problème d'agence entre les gestionnaires et les actionnaires de la banque. La dernière crise financière a montré les graves distorsions que l'innovation financière, a l'instar de la titrisation et ses produits (CDS, CDO, ABS.....), ont introduit et comment ils ont contribué à une prise de risque excessive, à travers une baisse des standards de risque, et IN FINI à la fragilisation du système bancaire (20)

Un autre effet négatif marquant lié aux innovations financière, est l'accroissement de la complexité et de l'opacité des systèmes financiers, des produits et des procédés financiers, ce qui accentue le fossé entre les inities (les institutions financières), et les non-initiés qui sont en majorité les petits épargnants qui n'ont ni la capacité d'analyser correctement les informations, ni celle de comprendre les mécanismes de fonctionnement.(21)

Un autre aspect négatif de l'innovation financière est que cette dernière peut surgir comme une réaction à la régulation financière en vigueur, l'émergence du marché de l'euro dollars en réaction au « $Q$ act » en est un bon exemple. Les récentes innovations financières ont, essentiellement, pour objectif le contournement de la règlementation et cela en transférant, pour des raisons de couts, les actifs du bilan au hors bilan. Et contrairement à la vision traditionnelle selon laquelle les innovations financières sont source d'efficience 
et de diversification des risques, les détracteurs de l'innovation financière voient en elle un moyen utilisé par les spéculateurs pour transférer le risque aux investisseurs naïfs qui ne savent pas réellement se qu'ils ont en leurs possessions, ou à ceux qui sont confient car ils croient, a tort, qu'ils sont couvet contre les risques si les choses tourne mal. Cette innovation financière généré , par la volonté de ses concepteurs, dans la but de contourner la réglementation n'améliore pas la croissance économique et la bonne allocation des ressources, mais au contraire, elle contribue à fragiliser le system financier et a déstabilisé l'économie.(22)

Les mutations financières, spécialement la déréglementation, la globalisation et le décloisonnement des activités financières ont permis la mise en place d'innovations financières qui captent la liquidité oisive fragilisent ainsi les structures financières puisque le nombre d'engagements augmente alors que la quantité de monnaie est inchangée. Cette situation augmente le risque d'une propagation en chaine de défauts. L'apparition de ce type d'innovations entraine également une hausse du ratio dette/valeur nette ce qui met en valeur le problème de la solvabilité des débiteurs.

Les comportements des institutions financières se modifient indépendamment de la volonté de la Banque centrale, ce qui entraine une augmentation substantielle du volume de l'activité économique que le système bancaire peut financer à travers l'émission d'innovations financières. Il suffit alors que les détenteurs de monnaie oisive achètent les innovations financières pour combler cet écart. Ce type de financement implique une réduction de la liquidité de l'économie au sens d'une réduction de la quantité de liquidités oisives ou du ratio liquidités/titres. Ce lien entre cycle des affaires et fragilité financière est appelée «la Financial Instability Hypothesis (FIH)».(23)

L'innovation financière est devenue un puissant facteur de croissance des marches financier, mais par la même occasion, un facteur de croissance des risques lies a l'immense effet levier que l'utilisation de ce genre de produits innovants permet, spécialement sur les marchés financiers non-règlementés tel que les marches de gré à gré, qui par la nature de leurs organisation, ne disposent pas de partie contrôle (chambre de compensation) contrairement aux marchés organisés .

\section{6-Les implications des CDS (Crédit default swaps) sur la stabilité}

financière systémique :

\section{A- Définition du contrat CDS :}

Les crédits default swaps(CDS) ou Swap de défaut de crédit, sont des innovations financières introduits en 1997 par JP Morgan, sont devenus la 
forme la plus répandue de dérivés de crédit, totalisant en 2008 un encours de 64 000 milliards de dollars en valeur notionnelle. Depuis le début de la crise financière, l'encours notionnel est descendu à 38000 milliards de dollars environ au premier semestre 2009, mais reste élevé. Il s'agit là de montants notionnels bruts ; selon les estimations de la Banque des règlements internationaux (BRI), l'exposition nette des principaux dealers sur le marché de CDS représentait 2900 milliards de dollars en juin 2009.

Parfois décrits comme des « instruments financiers complexes », les CDS sont en fait les plus simples de tous les dérivés de crédit. Un CDS est un contrat entre deux parties, un acheteur de protection et un vendeur de protection, aux termes duquel l'acheteur de protection est remboursé de la perte entraînée par un événement de crédit relatif à un instrument de référence à ce titre les CDS sont des produits financiers à mi-chemin entre un dérivé financier et un produit d'assurance.

L'événement de crédit peut être la défaillance de l'entité de référence, le défaut de paiement d'un coupon ou d'autres événements affectant l'entité tels que définis dans le contrat. En échange, l'acheteur de protection paie une prime, égale à un pourcentage annuel du montant notionnel, au vendeur de protection. La prime est cotée en points de base ou en points de pourcentage du notionnel, est appelée spread de CDS. Le paiement du spread est effectué sur une base annuelle, semestrielle ou trimestrielle à terme échu jusqu'à ce que l'échéance soit atteinte ou qu'un événement de crédit se produise.

L'acheteur de protection est ainsi protégé contre les pertes en cas de défaut de l'entité de référence. Si l'acheteur possède le titre de référence, le CDS représente une protection contre le défaut : cette forme d'" assurance contre le défaut » constituait la motivation initiale de sa création. Néanmoins le volume pharamineux du marché des CDS indique clairement qu'une part substantielle des contrats est de nature spéculative, à travers l'usage de CDS a nus, l'encours notionnel des CDS peut dans ce cas devenir plus important que l'encours de la dette totale de l'entité de référence(le sous-jacent).

Les CDS sont des produits dérivés de gré à gré et, comme tels, ils ne s'échangent pas sur des marchés organisés. Le marché des CDS est un marché animé par des intermédiaires appelés dealers ou " Assureurs Monoline " où un petit nombre de grandes institutions contrôlent l'essentiel des volumes, les dix dealers les plus importants traitent $90 \%$ du volume des transactions en montant notionnel brut. La concentration est encore plus élevée sur le marché américain, où les cinq plus grandes banques commerciales traitent plus de $90 \%$ des montants notionnels bruts. Dans une telle situation, le défaut d'un dealer majeur peut avoir d'importantes répercussions sur le reste du marché, car la concentration du marché sur quelques dealers interconnectés amplifie l'importance du risque de contagion qui peut dégénérer en crise systémique. 


\section{B- Les implications des CDS sur la stabilité Financière :}

Le développement du marché des $C D S$ relevait à l'origine d'une logique assurantielle permettant aux investisseurs institutionnels encadrés par des contraintes de rating de diversifier leurs placements, notamment en souscrivant une protection contre le défaut des municipalités et autres entités publiques. L'utilisation de $C D S$ s'est progressivement étendue à la couverture d'une gamme de créances toujours plus large (y compris les titres représentatifs de crédits hypothécaires) dans la cadre de la titrisation synthétique.

Le paroxysme de la complexité des opérations de titrisation a été atteint au début des années 2000, avec l'émergence des $C D O$ de $C D O$ ou $C D O^{2}$ et $C D O^{3}$. Il s'agit de montages comportant plusieurs couches de titrisation, c'est à dire plusieurs opérations de titrisation successives ou re-titrisation : à partir des $A B S$ et $C D O$ émis par deux véhicules spécialisés, un troisième $S P V$ émet des $C D O^{2}$. Ces titres sont donc adossés à des produits titrisés, qui eux même sont adossés au portefeuille d'actifs sous-jacents dans le cas d'une titrisation true sale ou à un contrat de swap dans le cas d'une titrisation synthétique. En réalité, les $\mathrm{CDS}$ sont devenues avec les $C D O, C D O^{2}$ et $C D O^{3}$ les symboles de la course folle du processus d'innovation financière visant à extraire davantage de profits, sans réelle création de valeur.

La finance structurée et les dérivés de crédit ont donc constitué des supports privilégiés pour les stratégies d'arbitrage réglementaire. La réglementation prudentielle promulgué avec les accords de Bale 1 en 1988, qui imposé un accroissement du coût en Fond propres associé à la détention d'actifs faiblement notés, a alors stimulé le marché des CDS. La demande pour des contrats de swap sur événement de crédit (CDS) portant sur des créances hypothécaires titrisés a explosé à partir de cette date.

$\mathrm{Ce}$ sont ces mêmes innovations particulièrement complexes qui ont largement contribué à la propagation du risque au cours de la crise financière de 2007-2008, car c'est la prise de conscience d'une sous-estimation généralisée du risque et l'impossibilité pour les intermédiaires d'évaluer l'exposition de leurs contreparties qui ont conduit à des dégradations des notations d'agence de plusieurs crans en une journée, provoquant le déclenchement des indemnisations contingentes prévues par certains $C D S$. .(24)

Il a été souvent reproché aux CDS d'entretenir l'instabilité financière et de générer du risque systémique. En interdisant en 2011 les transactions de CDS « nus » (achat de CDS sans détention de l'obligation rattachée à l'entité sous-jacente), l'autorité de régulation financière allemande, la BaFin, a déclaré que les évolutions des CDS mettaient en péril « la stabilité de l'ensemble du système financier ». Cette accusation est largement liée au rôle supposé de 
positions spéculatives des (CDS « nus »), dans la hausse des spreads de CDS d'entités en difficulté, rendant ainsi plus difficile leur accès aux marchés de la dette. L'exemple le plus récent étant celui de la Grèce. Selon les chiffres fournis par la Depository Trust and Clearing Corporation (DTCC), les positions de CDS sur la Grèce se sont élevées à 9,2 milliards de dollars (nets) en mars 2010, contre 7,4 milliards de dollars en 2009, et les spreads de CDS ont suivi de près la tendance des spreads obligataires en 2010

La spécialisation sur le marché des contrats CDS peut concentrer le risque et par la suite crée le risque systémique. Ce risque systémique peut être mal évalué et ignoré par les participants au marché pour une période de temps assez prolongée. Mais, une fois les conditions spécifiques s'installent, les défauts du système amplifient la crise et le risque systémique devient une réalité.

Le risque de contrepartie généré par le défaut d'importants vendeurs de protection, illustré par l'incapacité peut affecter de nombreux participants de marché et entraîner des effets de domino. Sur un marché de CDS, le défaut d'une entité entraîne des pertes non seulement pour ses contreparties mais également pour les vendeurs de CDS référencés sur cette entité. Si un vendeur de protection sur CDS ne dispose pas de réserves suffisantes pour couvrir ses engagements, l'événement de crédit relatif au sous-jacent entraîne également son défaut, ce qui élargit la portée de la contagion conduit à une hausse du processus du risque systémique. (25)

\section{Conclusion}

Le développement rapide et à grande échelle des innovations financières et un phénomène qui caractérise le système financier actuel, ce développement est intimement liés aux évènements économiques, politiques et technologiques qui ont secoués le monde a partir des années 70 . Les innovations financiers ont joué un rôle décisif dans le développement de des marchés financiers est en contribuer à ce titre au financement du progrès et de l'expansion de l'économie mondiale en adaptant les produit du financement aux besoins spécifiques des entreprises. Les innovations financières ont permis aussi aux acteurs économiques de disposer de moyens de gestion des risques que la nouvelle conjecture économique exigée.

Le développement des innovations financières utiles, c'est fait en parallèle avec le développement des innovations que l'ont peu qualifié d'inutile car ayants pour seul but la spéculation et le gain facile au détriment d'investisseurs crédules. La prolifération de ce type d'innovations financières est pour beaucoup à l' origine de l'instabilité des marchés financiers ces dernières années.

Les CDS ou credit default Swap sont une bonne illustration des dérives dangereuses qu'une innovation financière «utile », conçue initialement pour 

default swap (CDS).

garantir une meilleur distribution des risques, peu subir de la part des spéculateurs peu scrupuleux et se transformé ainsi en moyen de manipulation et de déstabilisation aux seules fins de réaliser des gains.

L'utilisation des CDS à nu lors de la crise de la dette souveraine Grecque en 2010 ou l'utilisation des CDS lors des opérations de titrisation synthétique des créances hypothécaires en 2007 , ont été des éléments aggravants des deux crises.

Remédier a cette situation passe par la mise en place d'une meilleure règlementation financière qui incitera les intermédiaires financières a plus de transparence, et qui impliquerais, entre autres, pour les innovations inutiles le payement de taxe sur les transactions financières de ce genre (taxe TOBIN) par leurs initiateurs. Ceci en plus de la proposition faite lors du G20de 2009 a Londres et qui consiste à transférer tous les transactions sur les produit dérives vers les marches organisés afin de mieux contrôlé les risques et leur implications sur la stabilité systémique..

\section{Références :}

(1) Capelle-Blancard .G, Couderc.N, Vandelanoite .S : « Les marchés

financier en fiches ». Ellipses Edition, Paris, 2004, page 30.

(2) Merton H .Miller: "Financial innovation: the last twenty years and the $\underline{\text { nexty }}$. Journal of financial and quantitative analysis, volume 21, No 4, December 1986, page 460.

(3) Schumpeter, J.A: "IThe Theory of Economic Development». Kluwer Academic, 1911, Page 61-116.

(4) Capelle- Blancard .G et al, Op.cit., page 31-32.

(5)Arnaud André-Jean : «Du droit des marchés financiers; entre modernité et mondialisation $"$

Paris LGDJ, 2004, page 176.

(6) Miskin. Frederic, $\mathrm{S}:$ «The economics of money, banking and financial market ". New York, harper Collins, 1995, page 233-245.

(7) Zumello .Christine: "L'innovation et les marchés financiers aux Etats-

Unis depuis les années 1970: une approche contrastée " . Revue LISA/LISA e-journal, Vol. IV- no 1, 2006 consulté le 13/12/2014, page 04.

http://lisa.revue.org/2385;DOI:10.4000/lisa.2385

(8) Kane Edward .J: "Interaction of financial and regulatory innovation"

American economic revue, Vol 78, no 2, 1988, page 329.

(9) Frame W. Scott, White Lawrence: «Empirical studies of financial innovation: Lots of Talk, Little action", Journal of economic literature, Vol 42, no 01, 2004, page 120.

(10) Capelle- Blancard .G et al, Op.cit., page 34. 
(11) Kane Edward .J, Op.cit. Page 332.

(12) Zumello .Christine, Op.cit., page 7.

(13) Granier Thiery, Jaffeux Corynne : «La titrisation : Aspects juridique et

financier " 2 . 2iem Edition, Collection Gestion, Edition Economica, 2004, Paris, Page 13.

(14) Granier Thiery, Jaffeux Corynne, Op.cit., page 158.

(15) Op.cit., page 169.

(16) Leathers .CG, Raines J.P: «The Schumpeterian role of financial innovations in the new economy's business cycle»", Cambridge journal of Economics, Vol 28, no 25, 2004, Page 669.

(17) Rose Peter.S; Marquise Milton .H: «Monev and capital markets», tenth Edition, Mc Graw-Hill Irwin, USA, 2008, Page 97-98.

(18) Van Horne J. C: «Of financial innovation and Excesses»». The journal of Finance, Vol.40, No 03, 1985 ,Page 626.

(19) Ritz Simon : "Les interactions entre innovations financières et

règlementaires : causes et conséquences de la crise financière ", mémoire de recherche en finance , Université de Lorraine, Nancy ,Année universitaire 2011 2012, Page 33-34.

(20) Beck Thorsten, Chen Tao, Lin Chen, Song Frank .M: « Financial Innovation: The Bright and the Dark Sides, 2012.page 3.

http://www.tcd.ie/Economics/assets/pdf/Bank_Financial_Innovation_Jan30 12.pdf

(21) Zumello .Christine, Op.cit., page 11-12.

(22) Beck Thorsten et al, Op.cit, page 4.

(23) Minsky Hyman .P: "The financial instability hypothesis $\boldsymbol{F I H}$ ", the Jerome levy institute of Bard College, Working paper No 74, USA, 1992, Page $1-10$.

http://www.levyinstitute.org/pubs/wp74.pdf

(24) Ritz Simon, Op.cit., page 81-84.

(25) Cont Rama : "Crédit Default swaps et stabilité financière", Revue de la stabilité financière, Banque de France, No 14, juillet 2010, Page 42-44.

https://www.banque-france.fr/publications/revue-de-la-stabilite-financiere.html 